\title{
CO 034 - FREE TESTOSTERONE (FT) IS INVERSELY RELATED TO FRAILTY IN HUMAN IMMUNODEFICIENCY VIRUS (HIV)-INFECTED MEN
}

S. De Vincentis ${ }^{1}$, M. C. Decaroli ${ }^{1}$, C. Diazzi ${ }^{1}$, F. Morini ${ }^{1}$, D. Bertani ${ }^{1}$, F. Fanelli ${ }^{2}$, M. Mezzullo ${ }^{2}$, D. Santi ${ }^{1}$, G. Tartaro ${ }^{1}$, E. Baraldi ${ }^{3}$, S. Tagliavini ${ }^{3}$, U. Pagotto ${ }^{2}$, G. Guaraldi ${ }^{4}$, V. Rochira ${ }^{1}$

${ }^{1}$ Unità di Endocrinologia, Dipartimento di Scienze Biomediche, Biometaboliche e Neuroscienze, Università di Modena e Reggio Emilia; Unità di Endocrinologia, Dipartimento di Specialità Mediche, Azienda Ospedaliero-Universitaria di Modena Modena, ${ }^{2}$ Unità di Endocrinologia e Centro di Ricerca Biomedica Applicata (CRBA), Dipartimento di Scienze Mediche e Chirurgiche, Università di BolognaOspedale Sant'Orsola-Malpighi, Bologna Bologna, ${ }^{3}$ Dipartimento di Medicina di Laboratorio e Anatomia Patologica, Azienda USL di Modena Modena, ${ }^{4}$ Clinica Metabolica Multidisciplinare, Unità di Malattie Infettive, Università di Modena e Reggio Emilia Modena

BACKGROUND: HIV-infection is associated to several age-related comorbidities, such as a premature decline of serum testosterone $(T)$. There is evidence about the relationship between health status, represented by frailty and comorbidities, and serum $T$ levels in general population, while only one previous retrospective study investigated it in HIV-infected men.

AIM: To investigate the association between frailty and gonadal status by assessing serum total T (TT) with Liquid Chromatography tandem Mass Spectrometry (LC-MS/MS) in a cohort of HIV-infected men.

METHODS: Prospective, cross-sectional, observational study on HIV-infected men (age $\leq 50$ years) with ongoing Highly Active Antiretroviral Therapy (HAART). Serum TT was assessed by the gold standard IDLC-MS/MS. Sex hormone-binding globulin (SHBG) was measured by chemiluminescent immunoassay. Free $\mathrm{T}$ (FT) was calculated by Vermeulen equation. Frailty was calculated through 38-items multimorbidity frailty index. Statistical analysis: Parameters were not normally distributed and MannWhitney $U$ test was used to compare continuous variables. Correlations were performed using linear regression models.

RESULTS: 315 consecutive HIV-infected men were enrolled (mean age $45.56 \pm 5.61$ years; average duration of HIV-infection $16.30 \pm 8.57$ years). 17 patients (5.4\%) had TT below $320 \mathrm{ng} / \mathrm{dL}$ and 31 patients (9.8\%) had calculated FT below $64 \mathrm{pg} / \mathrm{mL}$. Overall, 37 patients $(11.7 \%)$ had T deficiency defined by low TT levels and/or low FT. 56 patients (17.8\%) showed SHBG above the normal range (>71.4 $\mathrm{nmol} / \mathrm{L})$. Frailty score $(p=0.031)$, age $(p=0.001)$, duration of HIV-infection and of HAART $(p<0.0001)$ significantly differed between eugonadic and hypogonadic patients, while no difference was found for BMI $(p=0.209)$. FT inversely correlated with frailty score $\left(p=0.038, R^{2}=0.014\right)$, while TT did not $(p=0.235)$. At stepwise multivariate regression analysis, FT showed an inverse relation with age $\left(p<0.0001, R^{2}=0.150\right)$, years of infection $\left(-0.339, p<0.0001, R^{2}=0.125\right)$ and years of HAART $\left(-0.346, p<0.0001, R^{2}=0.117\right)$, but not with frailty score and $\mathrm{BMI}$ of patients.

CONCLUSIONS: To the best of our knowledge, this is the first properly-designed prospective study aiming to investigate the relationship between general health status and gonadal function in a cohort of HIV-infected men. FT is inversely related to frailty score, suggesting an impairment of gonadal function in those patients affected by more multimorbidities in this setting as well as in general population. At the same time, the age of patient and the duration of HIV-infection seem to be more potent predictive factors for serum FT levels than frailty score. In clinical practice it is important to check for testosterone in these patients due to frequent alterations of SHBG. 